\title{
Avaliação da reprodutibilidade da classificação de Schatzker revisada por Kfuri para as fraturas do planalto tibial
}

\section{Evaluation of the Reproducibility of the Schatzker Classification Reviewed by Kfuri for Tibial Plateau Fractures}

\author{
Henrique Mansur ${ }^{10}$ Victor Luiz Bastos Corrêa ${ }^{2}$ () \\ Marcello Teixeira Castiglia 4 (1) \\ 1 Serviço de Ortopedia e Traumatologia, Departamento de Ortopedia \\ e Traumatologia, Hospital Santa Helena, DF, Brasil \\ ${ }^{2}$ Departamento de Ortopedia e Traumatologia, Hospital das Forças \\ Armadas, Brasília, DF, Brasil \\ ${ }^{3}$ Serviço de Ortopedia e Traumatologia, Departamento de Ortopedia e \\ Traumatologia, Hospital Regional de Planaltina, Planaltina, DF, Brasil \\ ${ }^{4}$ Serviço de Ortopedia e Traumatologia, Departamento de Ortopedia \\ e Traumatologia, Hospital São Lucas, Ribeirão Preto, SP, Brasil
}

\author{
Bruno Abdo ${ }^{2(1)}$ Lucas Sacramento Ramos ${ }^{3}$
}

Rev Bras Ortop 2022;57(3):502-510.

\author{
Endereço para correspondência Henrique Mansur, Estudante de PhD \\ Departamento de Ortopedia e Traumatologia, Centro Clínico Cléo \\ Octávio SMHN, Quadra 2, Bloco B, Sala 305, Asa Norte, Brasília, \\ 70.710-146, DF, Brasil (e-mail: henrimansur@globo.com).
}

\section{Resumo}

\section{Palavras-chave}

- articulação do joelho

- classificação

- fraturas da tíbia

- reprodutibilidade dos testes

- traumatismos do joelho
Objetivo A classificação de Schatzker é a mais utilizada para as fraturas do planalto tibial. Kfuri et al. ${ }^{12}$ revisaram a classificação inicial de Schatzker descrevendo com mais detalhes o envolvimento do planalto tibial no plano coronal, permitindo uma melhor compreensão do padrão de fratura e um planejamento cirúrgico mais acurado. Os objetivos do presente estudo são avaliar a concordância inter-observador dessas classificações e avaliar a influência da experiência dos observadores na reprodutibilidade dos instrumentos.

Métodos Foi realizado um estudo observacional e retrospectivo, por meio da avaliação do estudo radiológico de 20 indivíduos adultos com fraturas do planalto tibial, incluindo radiografias e tomografia computadorizada (TC). As fraturas foram classificadas 1 vez por 34 examinadores com experiência variada (24 especialistas e 10 residentes em Ortopedia e Traumatologia), de acordo com a classificação de Schatzker e com a modificação proposta por Kfuri. O índice Kappa de Fleiss foi usado para verificar a concordância interobservadores. Resultados $\mathrm{O}$ índice de concordância inter-observador foi considerado moderado para a classificação de Schatzker $(\kappa=0,46)$ e leve para a modificação de Kfuri $(\kappa=0,30)$. A classificação de Schatzker apresentou concordância moderada, com $\mathrm{K}=0,52$ para residentes e $\mathrm{K}=0,45$ entre os especialistas. A classificação de Kfuri apresentou concordância leve com valores de Kappa para residentes e especialistas de 0,39 e 0,28 , respectivamente. recebido

03 de Setembro de 2020

aceito

03 de Novembro de 2020

Publicado on-line

Agosto 13, 2021
DOI https://doi.org/

$10.1055 / \mathrm{s}-0041-1729577$ ISSN 0102-3616.

\author{
(c) 2021. Sociedade Brasileira de Ortopedia e Traumatologia. All \\ rights reserved. \\ This is an open access article published by Thieme under the terms of the \\ Creative Commons Attribution-NonDerivative-NonCommercial-License, \\ permitting copying and reproduction so long as the original work is given \\ appropriate credit. Contents may not be used for commercial purposes, or \\ adapted, remixed, transformed or built upon. (https://creativecommons.org/ \\ licenses/by-nc-nd/4.0/) \\ Thieme Revinter Publicações Ltda., Rua do Matoso 170, Rio de \\ Janeiro, RJ, CEP 20270-135, Brazil
}




\begin{abstract}
Keywords

- knee joint

- classification

- tibial fractures

- reproducibility of results

- knee injuries

Objective The Schatzker classification is the most used for tibial plateau fractures. Kfuri et al. ${ }^{12}$ reviewed Schatzker's initial classification describing in more detail the involvement of the tibial plateau in the coronal plane, allowing a better understanding of the fracture pattern and a more accurate surgical planning. The objectives of the present study are to evaluate the interobserver agreement of these classifications and to evaluate the influence of the experience of the observer on the reproducibility of the instruments.

Methods An observational and retrospective study was conducted by evaluating the radiological study of 20 adult individuals with tibial plateau fractures, including radiographs and computed tomography (CT). The fractures were classified once by 34 examiners with varied experience ( 24 specialists and 10 residents in Orthopedics and Traumatology), according to the Schatzker classification and to the modification proposed by Kfuri. The Fleiss Kappa index was used to verify interobserver agreement. Results The interobserver agreement index was considered moderate for the Schatzker classification $(\kappa=0.46)$ and mild for the Kfuri modification $(\kappa=0.30)$. The Schatzker classification showed moderate agreement, with $\mathrm{k}=0.52$ for residents and $\mathrm{K}=0.45$ among specialists. The Kfuri classification showed mild agreement, with Kappa values for residents and specialists of 0.39 and 0.28 , respectively.

Conclusion The Schatzker classification and the classification modified by Kfuri presented moderate and mild interobserver agreement, respectively. In addition, the residents presented higher agreement than the specialists for the two systems studied.
\end{abstract}

Conclusão A classificação de Schatzker e a classificação modificada por Kfuri apresentaram concordância interobservadores moderada e leve, respectivamente. Além disso, os residentes apresentaram concordâncias superiores aos especialistas para os dois sistemas estudados.

\section{Introdução}

As fraturas do planalto tibial são lesões relativamente comuns, representando de 1 a $2 \%$ de todas as fraturas do corpo humano. ${ }^{1}$ Tais fraturas são decorrentes da aplicação de forças compressivas axiais combinadas ou não com estresse em varo ou em valgo na articulação do joelho. ${ }^{2}$ Fatores como o grau de energia aplicada, direção da força, posição do joelho e qualidade óssea dos pacientes são determinantes para a personalidade da fratura e seu grau de desvio. ${ }^{1,3}$ Por se tratar de uma fratura com acometimento articular, as fraturas do planalto tibial representam um risco para a função do joelho, objetivando-se, portanto, sempre que possível, a redução anatômica da superfície articular e a fixação estável para permitir a mobilização precoce. Assim, é fundamental o adequado entendimento das características da fratura para a definição da abordagem cirúrgica. ${ }^{4,5}$

As classificações são importantes ferramentas que podem definir o prognóstico e auxiliar na tomada de decisão do tratamento mais adequado. Pelo menos 38 sistemas foram descritos para classificar as fraturas do planalto tibial. ${ }^{6}$ Alguns estudos verificaram a reprodutibilidade dessas classificações, concluindo que tanto a análise inter- quanto intraobservador tem concordância de baixa a moderada. ${ }^{6-10}$ Um estudo prévio ressaltou que os sistemas disponíveis têm dificuldade em classificar as fraturas que envolvem a região posterior do planalto tibial. ${ }^{7}$ A classificação mais utilizada entre os cirurgiões ortopédicos é a de Schatzker, originalmente proposta em 1974. ${ }^{11}$ Este sistema é baseado em imagens biplanares, descrevendo seis tipos de fratura possíveis. Em 2018, Kfuri et al. ${ }^{12}$ revisaram a classificação de Schatzker adicionando a avaliação tridimensional com o auxílio da reconstrução tomográfica. Tal ferramenta propõe-se a descrever com mais detalhes o envolvimento do plano coronal, permitindo uma melhor compreensão do padrão da fratura e um planejamento cirúrgico mais adequado, uma vez que tal classificação ajuda na indicação da via de acesso mais adequada, em especial para as fraturas englobadas do tipo V e VI.

Estudos prévios avaliando a concordância de diferentes classificações das fraturas do planalto tibial variam muito em relação ao número de casos e de avaliadores. Por exemplo, um estudo prévio incluindo 3 observadores, ${ }^{13}$ e outro com 81 observadores ${ }^{14}$ reportaram resultados semelhantes com concordância leve para a classificação de Schatzker. Tais resultados poderiam representar que incluir poucos ou um número muito elevado de observadores poderia ocasionar redução na concordância das classificações. Até onde sabemos, somente Castiglia et al. ${ }^{15}$ avaliaram a reprodutibilidade da classificação de 
Schatzker ${ }^{11}$ e da classificação modificada por Kfuri et al. ${ }^{12}$ Neste estudo, 10 avaliadores classificaram 70 fraturas do planalto tibial, reportando concordância inter-observador moderada para ambos os sistemas, utilizando o coeficiente Kappa de Cohen.

Desta forma, para confirmar e expandir os resultados encontrados previamente, ${ }^{15}$ realizamos um estudo avaliando as fraturas do planalto tibial, incluindo um número maior de avaliadores. Os objetivos do presente estudo são avaliar a concordância inter-observador da classificação de Schatzker ${ }^{11}$ e da modificação proposta por Kfuri et al., ${ }^{12}$ e a influência da experiência técnica dos observadores no grau de confiabilidade das avaliações.

\section{Material e Métodos}

Trata-se de um estudo observacional e retrospectivo no qual foram analisadas imagens radiográficas e tomográficas obtidas de pacientes com fraturas da região proximal da tíbia tratadas no ano de 2019. Foram incluídos na pesquisa pacientes de ambos os gêneros, com idade $>18$ anos, com diagnóstico de fratura do planalto tibial em osso previamente sadio e submetidos a tratamento conservador ou cirúrgico. O estudo foi aprovado pelo Comitê de Ética em Pesquisa da Instituição sob o $n^{\circ} 15827019.0 .0000 .0023$.

O diagnóstico das fraturas foi realizado através de radiografias nas incidências anteroposterior (AP) e lateral, e tomografia computadorizada (TC) do joelho com reconstrução tridimensional e subtração do fêmur proximal e patela. Os critérios de exclusão foram: fratura prévia do planalto tibial, falta de um estudo radiológico completo ou estudo radiológico de qualidade considerada insuficiente.

Para cada paciente, foi criada uma sequência de slides de PowerPoint (Microsoft Corp., Redmond, WA, EUA) com um grupo de imagens composto por duas radiografias em projeções ortogonais, anteroposterior (AP) e lateral, além de imagens de TC nas projeções axial, sagital, e coronal, e da reconstrução tridimensional (3D) de imagens tomográficas da tíbia proximal. O conjunto de slides dos pacientes incluídos formou uma apresentação que foi apresentada aos avaliadores.

Os avaliadores eram médicos ortopedistas com diferentes níveis de experiência na área, sendo 24 membros da Sociedade Brasileira de Ortopedia e Traumatologia (SBOT), considerados "especialistas," e 10 médicos residentes (1 estagiário em cirurgia de joelho; 3 residentes do $3^{\circ}$ ano, 4 do $2^{\circ}$ ano, e 2 do

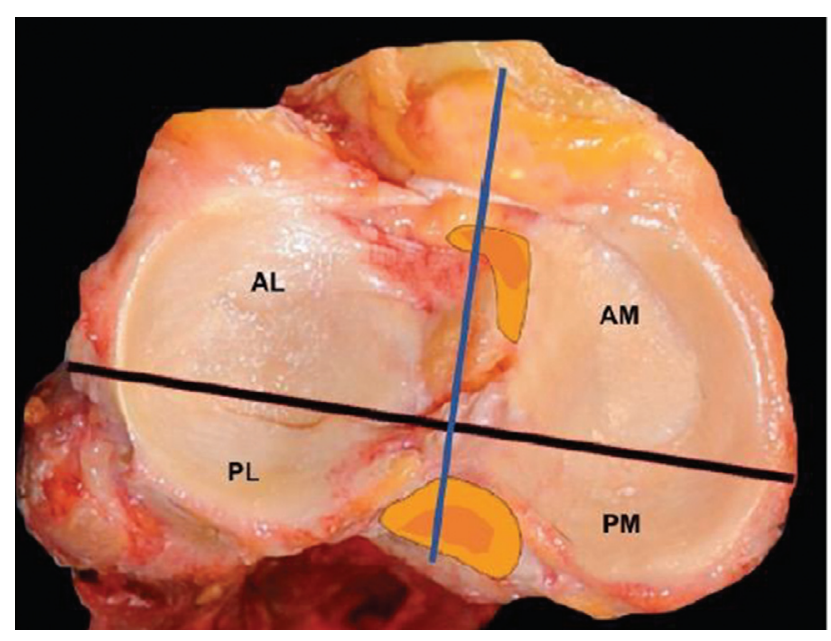

Fig. 2 Fotografia de peça cadavérica do planalto tibial com o equador virtual traçado dividindo a superfície articular em dois hemisférios, posterior e anterior. Legenda: $\mathrm{AL}$ - anterolateral, $\mathrm{PL}$ - pósterolateral, AM - anteromedial, PM - posteromedial. (Reproduzido de Castiglia MT. ${ }^{15}$ Complementação tomográfica da classificação de Schatzker para as fraturas do planalto tibial [tese]. Ribeirão Preto. Faculdade de Medicina de Ribeirão Preto; 2017).

$1^{\circ}$ ano de Ortopedia e Traumatologia). Para aferir a concordância inter-observadores, os 34 avaliadores classificaram as fraturas uma única vez pelos dois sistemas descritos, após uma breve explanação e demonstração de figuras ilustrativas com as classificações de Schatzker ${ }^{11}$ e sua modificação proposta por Kfuri et al. ${ }^{12}$ Foi permitido aos participantes consultar a qualquer momento as classificações (- Figuras 1 e $\mathbf{2}$ ). 0 grau de influência da experiência dos observadores foi avaliado por meio da concordância das avaliações dos especialistas e dos residentes, isoladamente.

\section{Sistemas de Classificação}

A classificação de Schatzker ${ }^{11}$ descreve seis tipos de lesão: Tipo I, cisalhamento do planalto lateral; Tipo II, cisalhamento associado a depressão do planalto lateral; Tipo III, depressão isolada do planalto lateral; Tipo IV, fratura isolada do planalto medial; Tipo V, fratura bicondilar com alguma preservação da continuidade óssea com a diáfise; Tipo VI, fratura bicondilar dissociada da diáfise (- Figura $\mathbf{1}$ ).

Na classificação modificada por Kfuri et al., ${ }^{12}$ os seis tipos descritos por Schatzker permanecem, porém foram adicionados
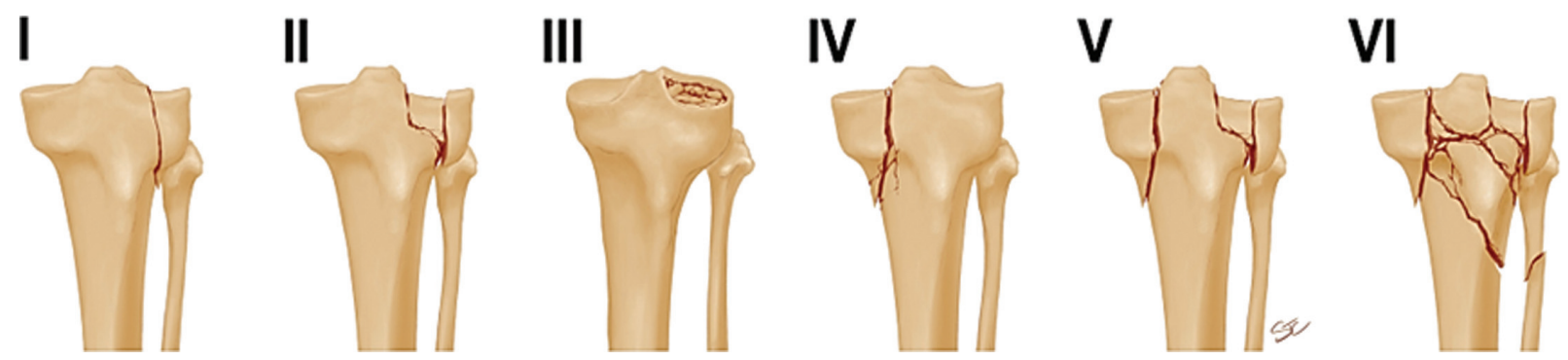

Fig. 1 Classificação de Schatzker para as fraturas do planalto tibial. 
os modificadores "A" para anterior e "P" para posterior, delimitando quatro áreas anatômicas distintas, especificamente: anterior medial, anterior lateral, posterior medial e posterior lateral. A divisão da superfície articular do planalto tibial é feita por um equador virtual, que divide o planalto tibial em dois hemisférios, posterior e anterior. As referências anatômicas para a criação deste equador virtual são, lateralmente, a inserção do ligamento colateral lateral na cabeça da fíbula e, medialmente, a linha de inserção posterior das fibras do ligamento colateral medial superficial junto à crista tibial medial (-Figura 2).

\section{Análise Estatística}

A análise estatística dos resultados obtidos foi feita utilizando o teste Kappa de Fleiss para avaliar a concordância interobservadores para as classificações de Schatzker ${ }^{11}$ e para sua modificação por Kfuri et al. ${ }^{12}$ Foram igualmente analisados os resultados das avaliações por grupo de observadores, especialistas e residentes. O coeficiente de Kappa é o método de análise estatística mais comum, e está presente em muitos artigos que avaliam a concordância entre dois examinadores (ou dois métodos). Fleiss ${ }^{16}$ propôs uma extensão do Kappa para quando há mais de dois examinadores (ou métodos). Assim, o uso do coeficiente Kappa de Fleiss é considerado o mais apropriado quando nos defrontamos com a situação na qual múltiplos examinadores estão envolvidos ou avaliações são feitas, e quando a escala avaliada apresenta muitas categorias. Seu valor varia de +1 (concordância perfeita), passa por 0 (concordância igual ao acaso) e vai até - 1 (discordância completa). ${ }^{17,18}$

Não há definições quanto aos níveis de concordância aceitos, mas Landis et al. ${ }^{19}$ propuseram a seguinte interpretação: resultados entre 0 e 0.19 apresentam concordância pobre; entre 0.2 e 0.39 , concordância leve; entre 0.4 e 0.59 , concordância moderada; entre 0.6 e 0.79 , concordância substantiva; e um valor $>0,80$ é considerado concordância quase perfeita. 0 critério de determinação de significância adotado foi o nível de 5\%. A análise estatística foi processada pelo software estatístico SAS System, versão 6.11 (SAS Institute, Inc., Cary, NC, EUA).

\section{Resultados}

0 índice de concordância interobservador foi considerado moderado para a classificação de Schatzker $(\mathrm{K}=0.46)$ e leve para a modificação de Kfuri ( $\mathrm{K}=0.30)$.

Ao analisarmos as classificações de acordo com o nível de experiência do avaliador (especialistas versus residentes), observamos índices de concordância com interpretações semelhantes, porém com valores maiores no grupo de residentes. A classificação de Schatzker apresentou concordância moderada, $\operatorname{com~} \mathrm{K}=0.52$ entre residentes e $\mathrm{K}=0.45$ entre os especialistas. A classificação de Kfuri apresentou concordância leve, com valores de Kappa entre residentes e especialistas de 0.39 e 0.28 , respectivamente (-Tabela 1). Na - Tabela 2, estão descritos os resultados das avaliações das fraturas do planalto tibial dos 20 pacientes, pelos 34 examinadores.

\section{Discussão}

Diversos estudos investigaram a confiabilidade das classificações das fraturas envolvendo o planalto tibial na tentativa de se definir qual sistema tem a capacidade de auxiliar na tomada de decisão do tratamento. ${ }^{6-12}$ Entretanto, são reportados valores de concordância considerados baixos a moderados para a análise inter- e intraobservador. ${ }^{6-10}$ Os principais achados do presente estudo demonstram que a classificação de Schatzker apresentou concordância interobservador moderada, enquanto a classificação de Schatzker modificada por Kfuri apresentou concordância leve. Além disso, diferentemente do que esperávamos, os residentes apresentaram maior concordância interobservador para as duas classificações.

A recente classificação de Schatzker modificada por Kfuri et al. ${ }^{12}$ tem como proposta detalhar o padrão das fraturas do planalto tibial, permitindo uma melhor compreensão e planejamento cirúrgico através da escolha da via de acesso mais adequada. Em nosso estudo, as classificações de Schatzker e a proposta por Kfuri apresentaram valores de Kappa da concordância interobservador interpretados como moderada e leve, respectivamente. Estes resultados confirmam parcialmente e expandem os achados do estudo realizado por Castiglia et al. ${ }^{15}$ No único estudo que avaliou a concordância da classificação de Kfuri, os autores relataram valores de Kappa superiores aos nossos, com coeficiente de concordância substancial e moderado para os mesmos instrumentos, respectivamente. Acreditamos que esta diferença se deveu a algumas razões: tamanho de amostra com 20 casos, diferença de formação e experiência dos avaliadores e inclusão somente imagens de raio-X (RX) e de TC das fraturas, enquanto no outro estudo foram disponibilizados aos observadores, além de imagens de RX, vídeos com todos os planos e da reconstrução da TC. Ambas as situações podem ter atuado diminuindo a concordância tanto inter- quando intraobservador. 0 instrumento descrito de Kfuri, apesar de permitir uma avaliação mais detalhada das fraturas, oferece um maior número de possibilidades de classificações, aumentando sua complexidade. ${ }^{12,15}$ Em concordância com nosso estudo, Castiglia et al. ${ }^{15}$ descreveram confiabilidade superior da classificação de Schatzker em relação a sua modificação. Estudos prévios observaram que sistemas mais complexos para a classificação de fraturas apresentam menor concordância interobservador pelo maior número de opções para os avaliadores..$^{20,21}$ Observamos maior concordância nos casos mais complexos, envolvendo as quatro áreas anatômicas do planalto tibial, bem como nas fraturas menos complexas, envolvendo uma única área. Entretanto, pelo limitado tamanho amostral do presente estudo, esta informação deve ser considerada com restrições.

A classificação de Schatzker ${ }^{11}$ é a mais utilizada entre os cirurgiões ortopédicos; consequentemente, a maioria dos avaliadores possui familiaridade com ela. Levando em consideração esta classificação, observamos uma concordância moderada entre os observadores. Tais resultados são suportados pela literatura, que apresenta resultados semelhantes, com concordância de leve a moderada. ${ }^{6-10,13,21-24}$ Uma vez que a TC é indispensável para a aplicação da classificação de Kfuri, em nosso trabalho, os avaliadores utilizaram as imagens 


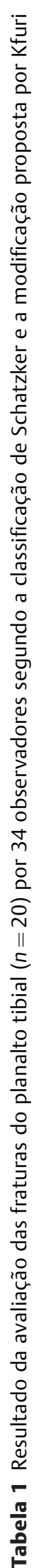

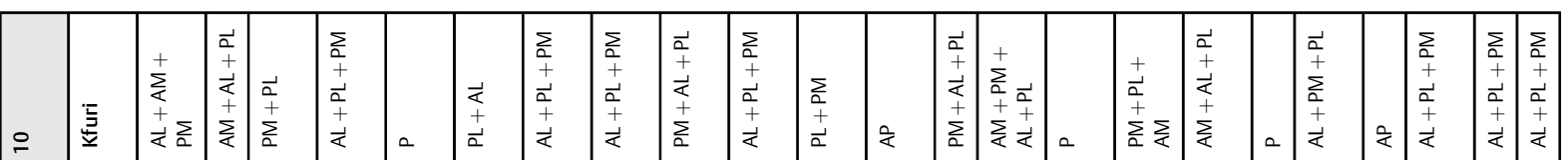

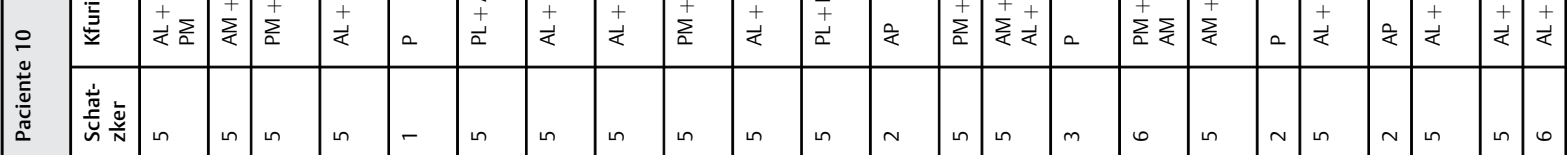

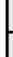

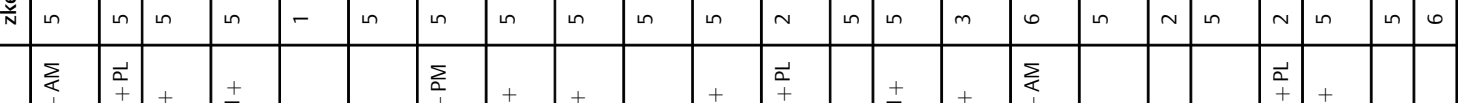

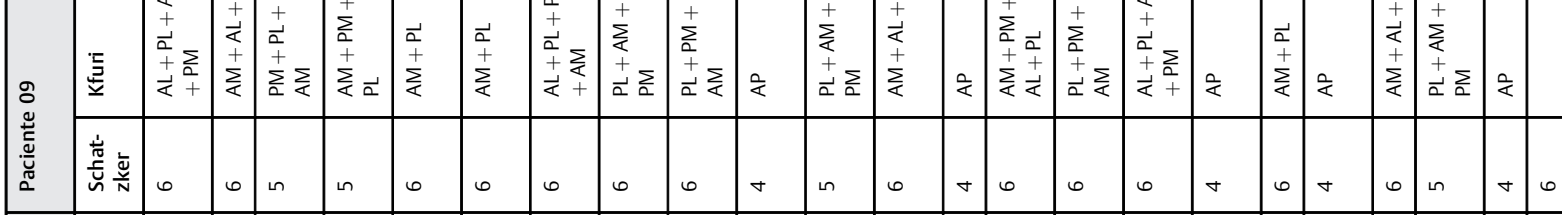

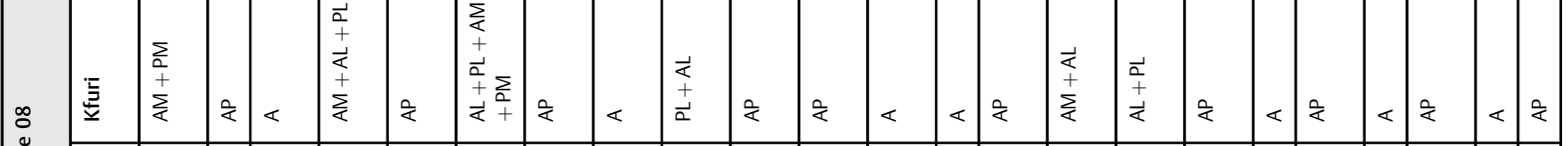

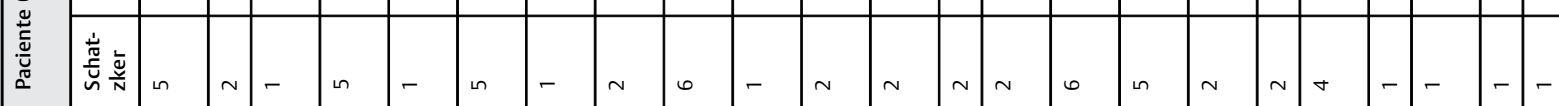

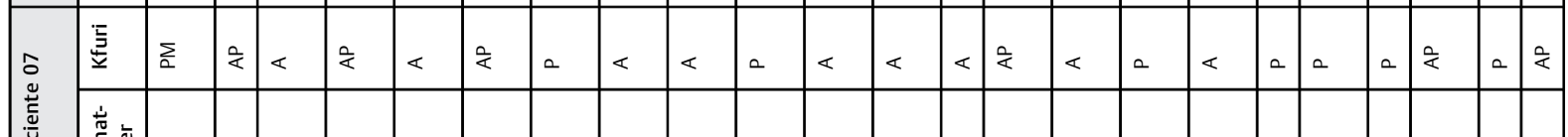

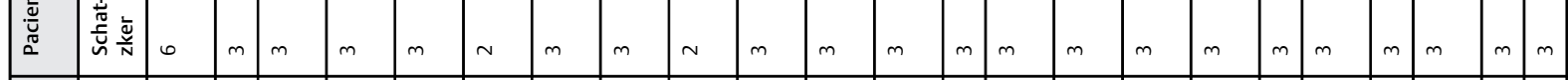

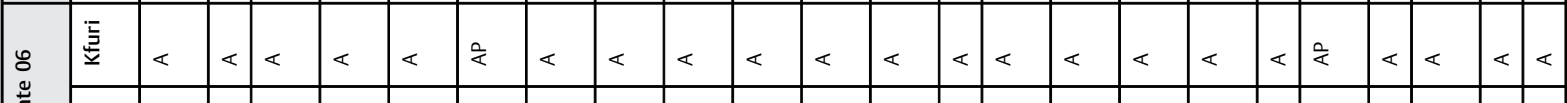

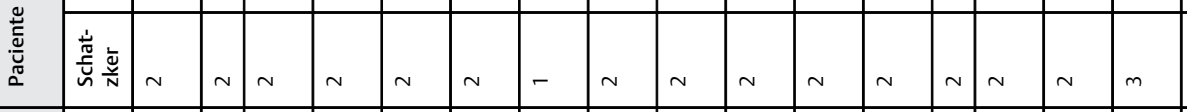

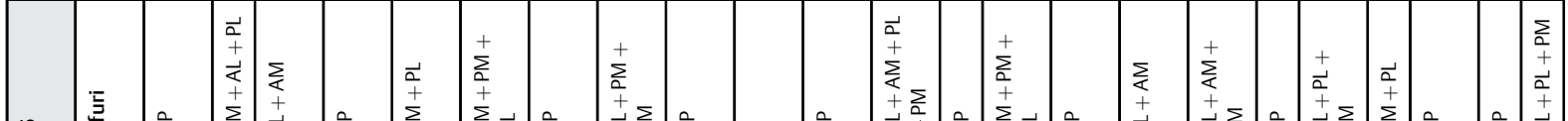

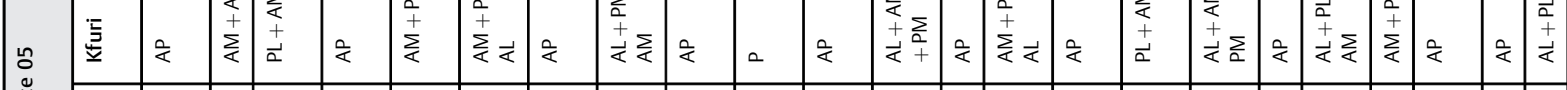

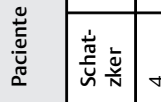

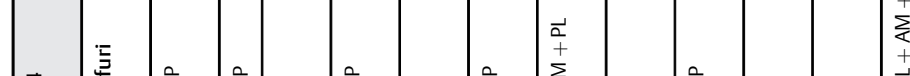

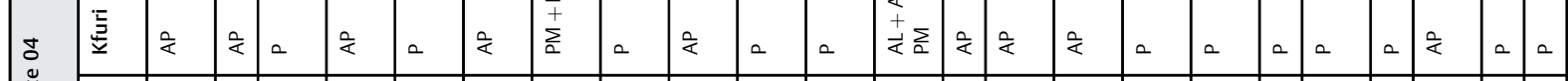

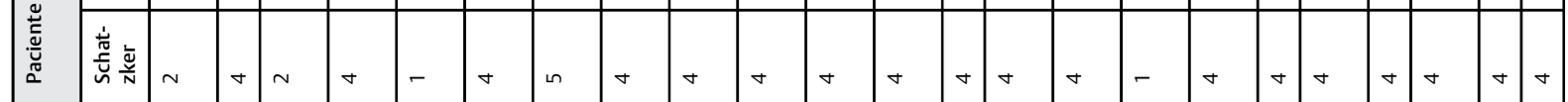

m

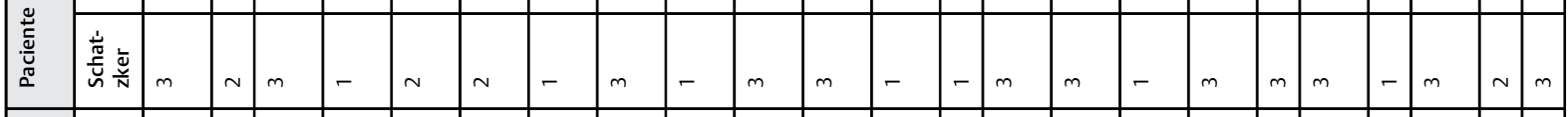

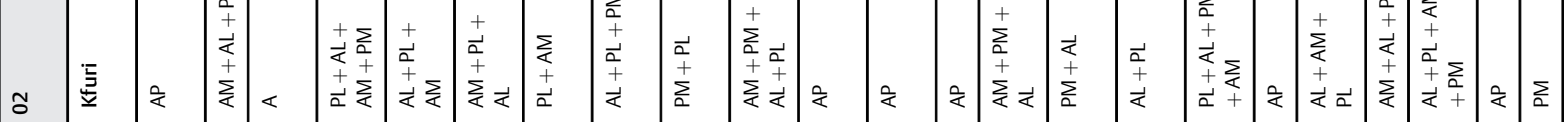

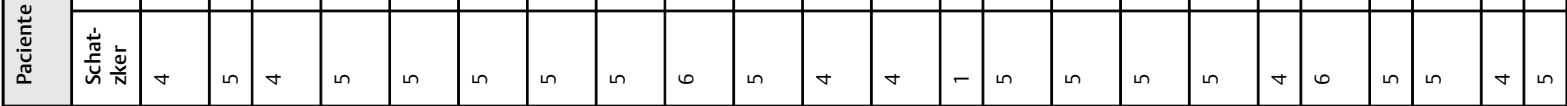

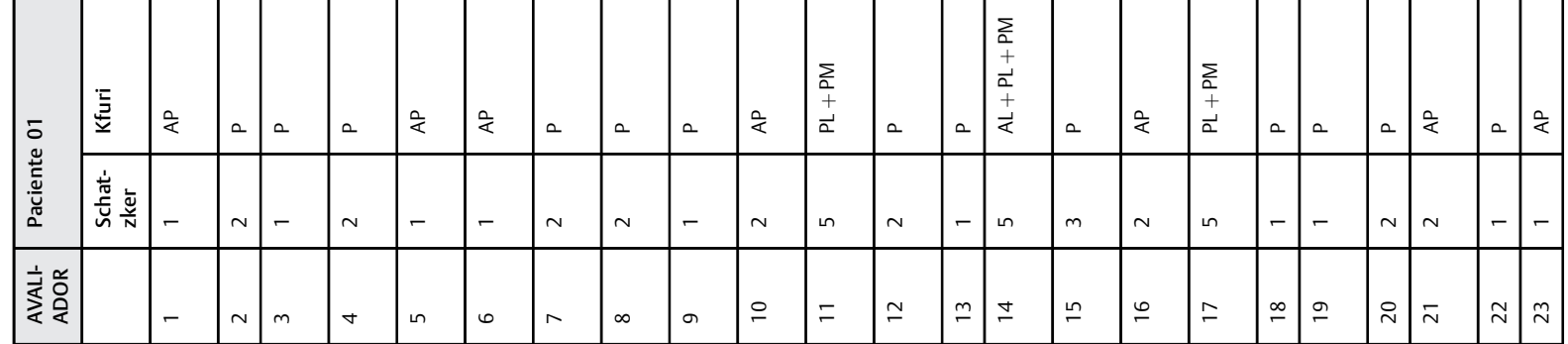




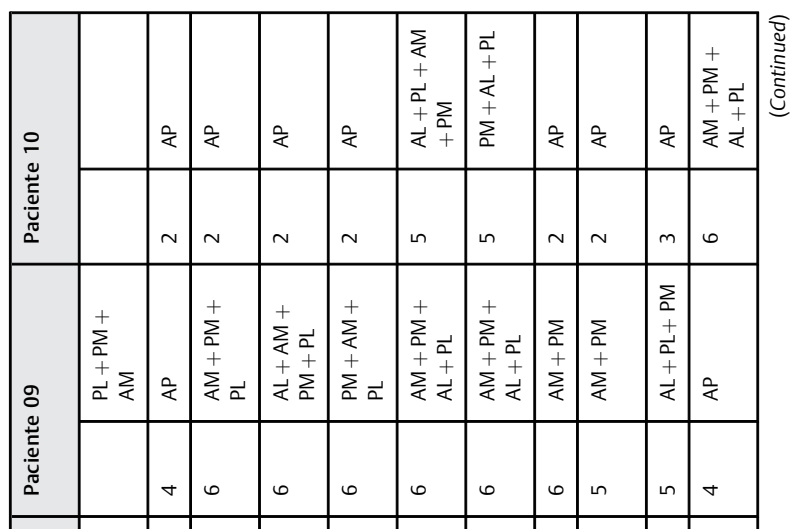

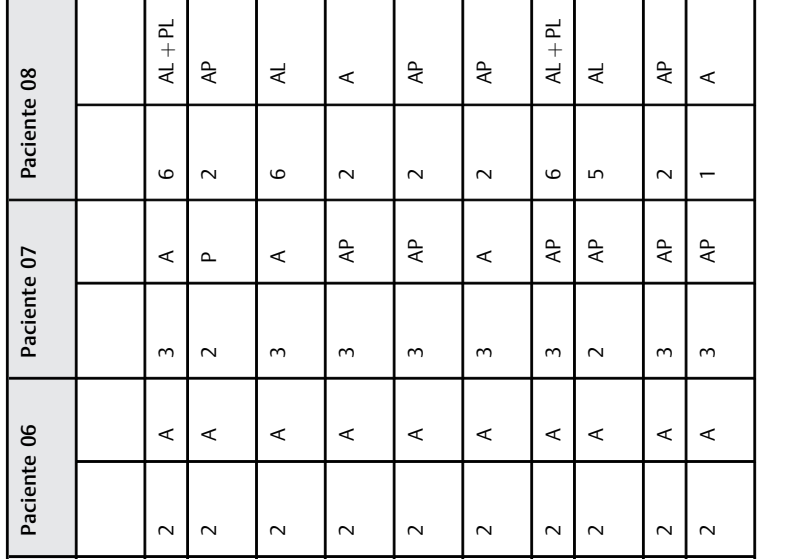

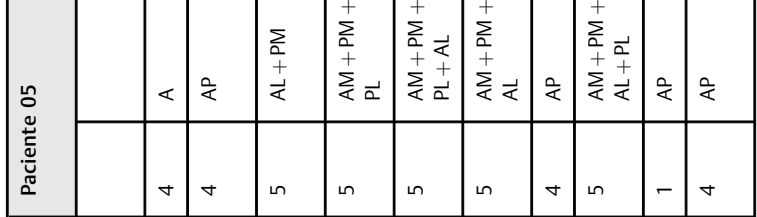

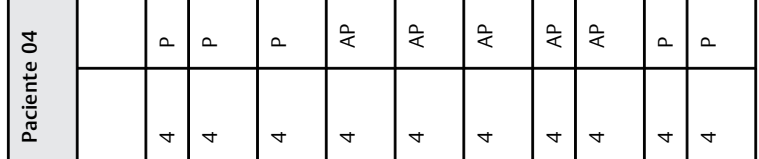

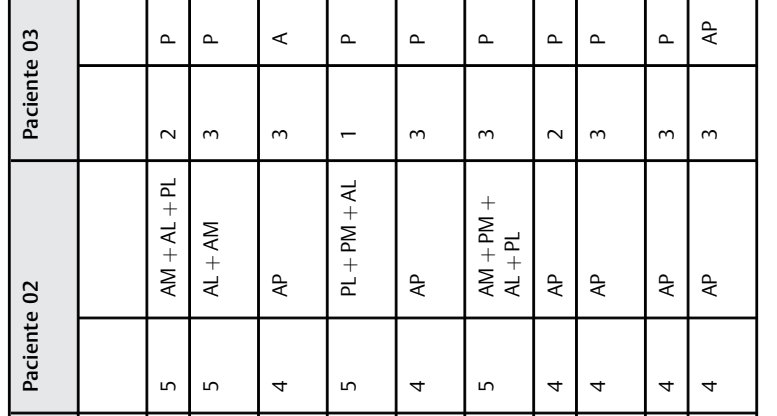

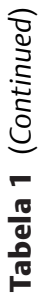

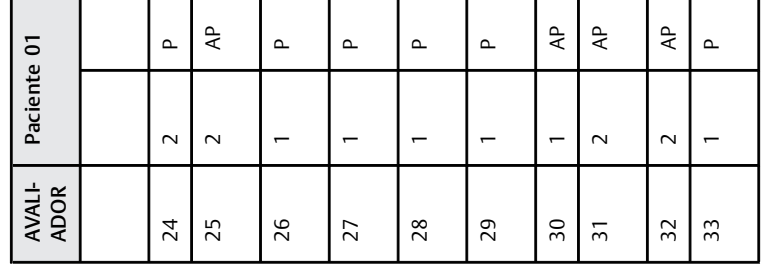

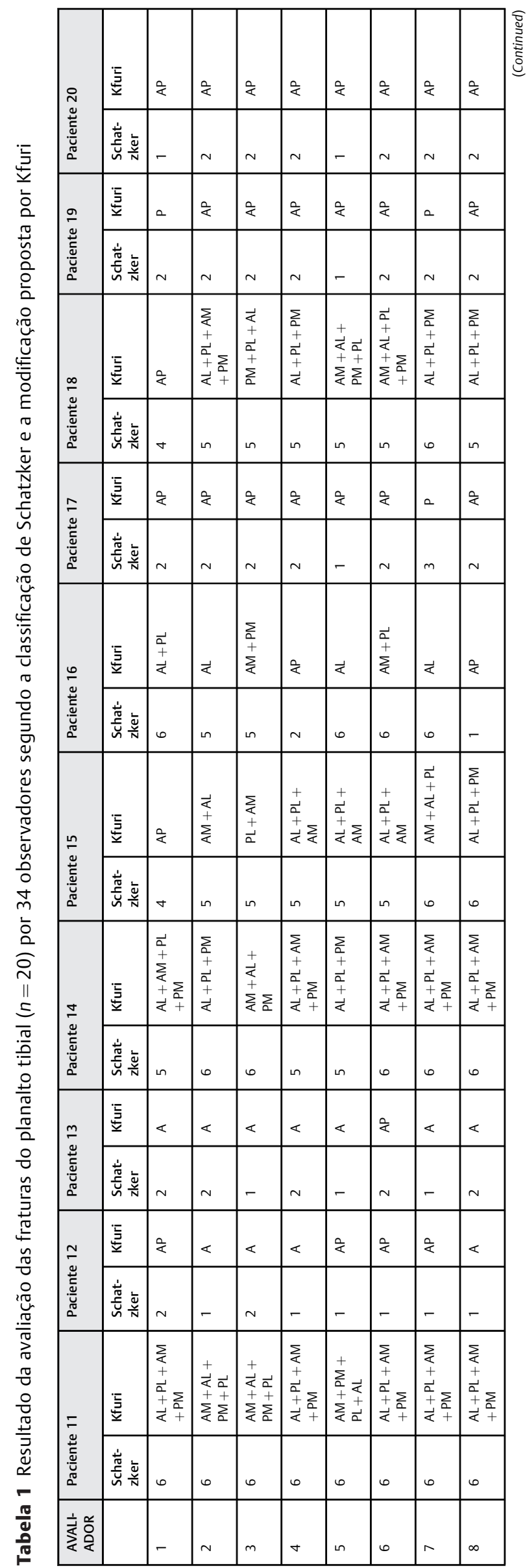




\begin{tabular}{|c|c|c|c|c|c|c|c|c|c|c|c|c|c|c|c|c|c|c|c|c|c|c|}
\hline \multirow{2}{*}{ 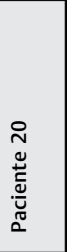 } & $q$ & \begin{tabular}{|l}
$\frac{\Sigma}{a}$ \\
+ \\
$\vec{a}$ \\
+ \\
$+\vec{\alpha}$
\end{tabular} & q & $q$ & $q$ & $q$ & $q$ & $\mid<$ & $q$ & $q$ & $q$ & q & $q$ & 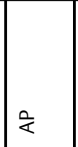 & $q$ & $q$ & $<$ & $q$ & 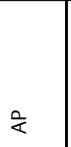 & q & $q$ & 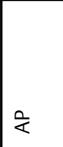 \\
\hline & $\sim$ & in & m & - & N & N & $\sim$ & - & $\sim$ & - & - & - & - & - & $\sim$ & $\sim$ & - & $\sim$ & $\sim$ & $\sim$ & $\sim$ & $\sim$ \\
\hline \multirow[t]{2}{*}{ 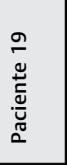 } & a & $q$ & $q$ & $q$ & $q$ & $q$ & $\gtrless$ & a & 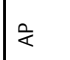 & a & a & 安 & o & a & $\%$ & 安 & a & \& & a & 安 & \& & \& \\
\hline & $\sim$ & $\sim$ & m & $\sim$ & $\sim$ & $\sim$ & $\sim$ & $m$ & $\sim$ & $m$ & $\sim$ & $\sim$ & $\sim$ & $\sim$ & $\sim$ & $\sim$ & $\sim$ & $\sim$ & $\sim$ & $\sim$ & $\sim$ & $\sim$ \\
\hline \multirow{2}{*}{ 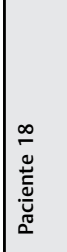 } & \begin{tabular}{|l}
+ \\
+ \\
0 \\
0 \\
+ \\
$\sum_{0}^{+} \sum^{+}$
\end{tabular} & 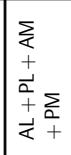 & 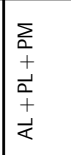 & 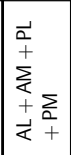 & \begin{tabular}{|l} 
\\
$\vec{a}$ \\
+ \\
$\sum_{a}$ \\
0 \\
$\dot{\alpha}$ \\
$\vec{\alpha}$
\end{tabular} & 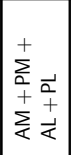 & 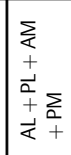 & 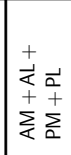 & 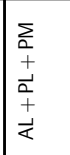 & 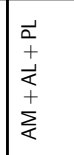 & 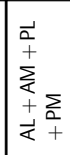 & 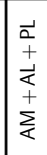 & 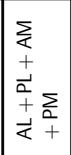 & \begin{tabular}{|l|}
$\sum_{a}$ \\
+ \\
$+\vec{a}$ \\
+ \\
+ \\
$\overrightarrow{<}$
\end{tabular} & 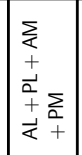 & 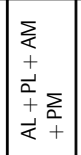 & 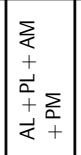 & \begin{tabular}{|l}
$\vdots$ \\
$\vdots$ \\
+ \\
$\dot{\alpha}$
\end{tabular} & 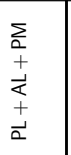 & $\begin{array}{l}\sum_{a} \\
+ \\
+\vec{a} \\
\underline{a} \\
+ \\
\overrightarrow{<}\end{array}$ & $\begin{array}{l}\sum_{a} \\
+ \\
+ \\
a \\
+ \\
\overrightarrow{<} \\
\overrightarrow{<}\end{array}$ & $\begin{array}{l}+ \\
\sum_{0}^{+} \\
+\vec{a} \\
\sum_{x}^{+}+\frac{1}{x}\end{array}$ \\
\hline & 0 & in & in & 0 & 0 & 0 & 0 & in & in & in & in & in & 0 & 0 & 0 & in & in & in & in & in & in & in \\
\hline \multirow{2}{*}{ 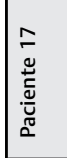 } & 安 & $\gtrless$ & \& & \& & $<$ & \& & $q$ & $\mid<$ & \& & $q$ & $q$ & $q$ & $q$ & 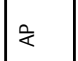 & \& & $<$ & $q$ & 安 & 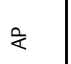 & i & 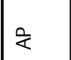 & \& \\
\hline & - & - & $\sim$ & - & - & - & - & - & $\sim$ & - & $\sim$ & - & - & - & - & - & - & - & - & N & N & - \\
\hline \multirow[b]{2}{*}{ 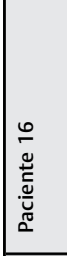 } & $\vec{\varangle}$ & $\ll$ & $\begin{array}{l}\sum \\
\sum \\
+ \\
\vec{\alpha}\end{array}$ & $\vec{\alpha}$ & 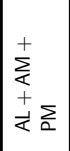 & ব & 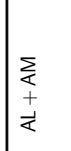 & $\ddot{<}$ & $\begin{array}{l}\Sigma \\
\Sigma \\
+ \\
+\vec{a} \\
+ \\
+ \\
\dot{\tau}\end{array}$ & a & $\begin{array}{l}\vec{a} \\
+ \\
\vec{\tau}\end{array}$ & $\rightleftarrows$ & $q$ & $\mid<$ & $\vec{\varangle}$ & $\vec{\varangle}$ & 安 & a & $\vec{\varangle}$ & $<$ & \begin{tabular}{|l}
$\vdots$ \\
$\vdots$ \\
+ \\
$\vec{\alpha}$
\end{tabular} & $\mid \begin{array}{l}\vec{a} \\
+ \\
\vec{\alpha}\end{array}$ \\
\hline & 0 & - & in & 0 & 0 & 0 & 0 & in & ڤn & - & 0 & 0 & - & - & 0 & in & ब & \% & 0 & \% & in & 0 \\
\hline \multirow[t]{2}{*}{ 은 } & 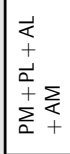 & \begin{tabular}{|l}
$\sum_{a}$ \\
+ \\
$\vec{a}$ \\
+ \\
$\dot{\alpha}$ \\
\multirow{2}{*}{}
\end{tabular} & $\begin{array}{l}\sum_{x} \\
+ \\
\dot{\alpha}\end{array}$ & $\begin{array}{l}\vec{a} \\
+ \\
+ \\
\vec{x} \\
+ \\
\sum_{\gtrless}^{+}\end{array}$ & \begin{tabular}{|l}
$\frac{\sum}{\alpha}$ \\
\pm \\
\\
\end{tabular} & 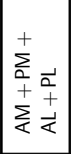 & $\mid \begin{array}{l}\sum_{0} \\
+ \\
+ \\
\sum_{\alpha}^{+}+ \\
a\end{array}$ & 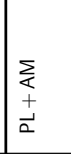 & 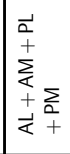 & $\begin{array}{l}\vec{a} \\
\sum^{+} \\
\sum_{<}\end{array}$ & $\begin{array}{l}\sum_{0} \\
+ \\
\sum^{+} \\
\end{array}$ & 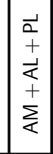 & $\begin{array}{l}\sum_{x} \\
\pm \\
\vec{a} \\
+\frac{\Sigma}{a} \\
+\frac{a}{\alpha}+\end{array}$ & \begin{tabular}{|l}
$\sum_{a}$ \\
+ \\
$+\vec{a}$ \\
+ \\
$\dot{\alpha}$ \\
\multirow{2}{*}{}
\end{tabular} & 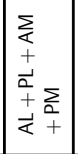 & 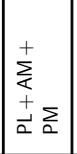 & 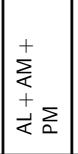 & 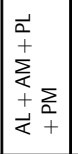 & $\begin{array}{l}\sum_{a}^{1} \\
\sum_{i}^{+} \\
a\end{array}$ & 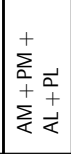 & \begin{tabular}{|l|}
+ \\
$\vec{a}$ \\
+ \\
$\sum_{<}^{+}$ \\
\end{tabular} & 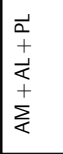 \\
\hline & 0 & in & in & in & in & 0 & 0 & 0 & 0 & in & 乞n & in & 0 & 0 & 0 & 0 & 0 & in & in & in & in & in \\
\hline \multirow{2}{*}{ 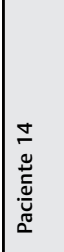 } & 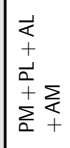 & 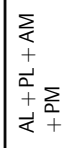 & $\begin{array}{l}\sum_{\alpha} \\
+ \\
\vec{a} \\
+\frac{\Sigma}{a} \\
+\frac{a}{\alpha}+\end{array}$ & 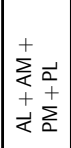 & 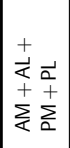 & 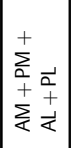 & $\mid \begin{array}{l}\sum_{a}^{1} \\
+\vec{a} \\
\sum^{+}+ \\
\sum_{\alpha}^{+}\end{array}$ & 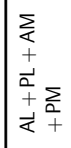 & 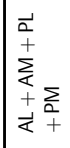 & 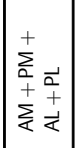 & 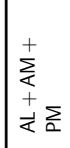 & 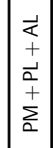 & $\begin{array}{l}\sum \\
\sum \\
+ \\
\vec{a} \\
+\frac{\Sigma}{a} \\
+\frac{a}{\alpha}+\end{array}$ & 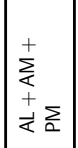 & 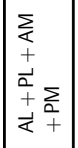 & $\left|\begin{array}{l}\sum_{x}^{+} \\
+ \\
\dot{z} \Sigma \\
\dot{\alpha}\end{array}\right|$ & 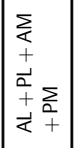 & 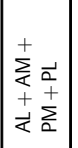 & 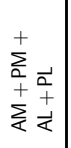 & 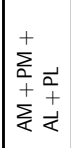 & 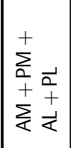 & 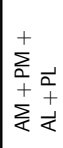 \\
\hline & 0 & 0 & 0 & 0 & 0 & 0 & 0 & 0 & 6 & 6 & 0 & 0 & 0 & 0 & 6 & 0 & 0 & in & in & 0 & 0 & in \\
\hline \multirow[t]{2}{*}{$\underline{m}$} & $\ll$ & $<$ & $<$ & $<$ & $<$ & $<$ & $\ll$ & $<$ & $<$ & $<$ & $\ll$ & $<$ & $\&$ & $\ll$ & \& & $<$ & $<$ & $<$ & $\ll$ & $<$ & $<$ & $\ll$ \\
\hline & $\sim$ & $\sim$ & m & $\sim$ & - & $m$ & $\sim$ & $\sim$ & $\sim$ & $\sim$ & $\sim$ & m & $\sim$ & - & - & $\sim$ & - & - & $\sim$ & m & m & m \\
\hline \multirow{2}{*}{ 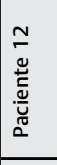 } & $<$ & $<$ & $<$ & $<$ & $\varangle$ & $<$ & $\varangle$ & $\varangle$ & $<$ & $<$ & \& & $<$ & $<$ & $\approx$ & 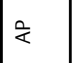 & $<$ & \& & $<$ & $\ll$ & $q$ & $<$ & $<$ \\
\hline & - & - & - & - & - & - & $\sim$ & $\sim$ & $\sim$ & - & $\sim$ & - & $\sim$ & - & - & - & - & - & m & $\sim$ & $\sim$ & - \\
\hline \multirow{2}{*}{ 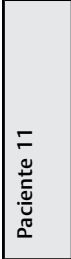 } & 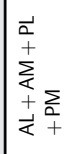 & 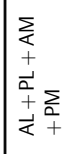 & 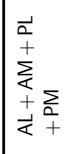 & 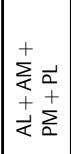 & 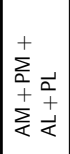 & 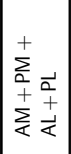 & 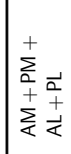 & 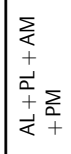 & 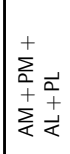 & 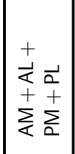 & 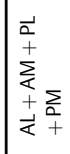 & $\begin{array}{l}\Sigma_{a} \\
+ \\
+\vec{a} \\
+ \\
\dot{\vec{\alpha}}\end{array}$ & $\begin{array}{l}\sum \\
\sum \\
+ \\
\vec{a} \\
+\frac{2}{a} \\
+\frac{a}{\alpha}+\end{array}$ & 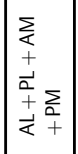 & 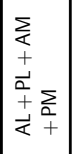 & 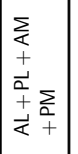 & 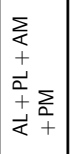 & 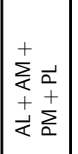 & $\begin{array}{l}+ \\
\vec{x} \\
+\vec{a} \\
+\sum_{x}^{+} \\
\sum_{a}^{+}\end{array}$ & 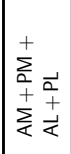 & 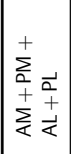 & 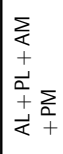 \\
\hline & 0 & 6 & 0 & 0 & 0 & 0 & 0 & 0 & 0 & 0 & 0 & 0 & 0 & 0 & 0 & 0 & 0 & 0 & in & 0 & 0 & in \\
\hline 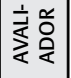 & $\sigma$ & 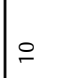 & $=$ & $\simeq$ & $m$ & \pm & $\stackrel{n}{\underline{n}}$ & $\stackrel{\circ}{\circ}$ & $=$ & $\stackrel{\infty}{\simeq}$ & 9 & 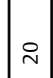 & $\bar{N}$ & $\approx$ & $\approx$ & $\mid \mathrm{N}$ & $\stackrel{\sim}{\sim}$ & $\stackrel{\sim}{i}$ & $\AA$ & $\stackrel{\infty}{\sim}$ & Iิ & $\ddot{m}$ \\
\hline
\end{tabular}




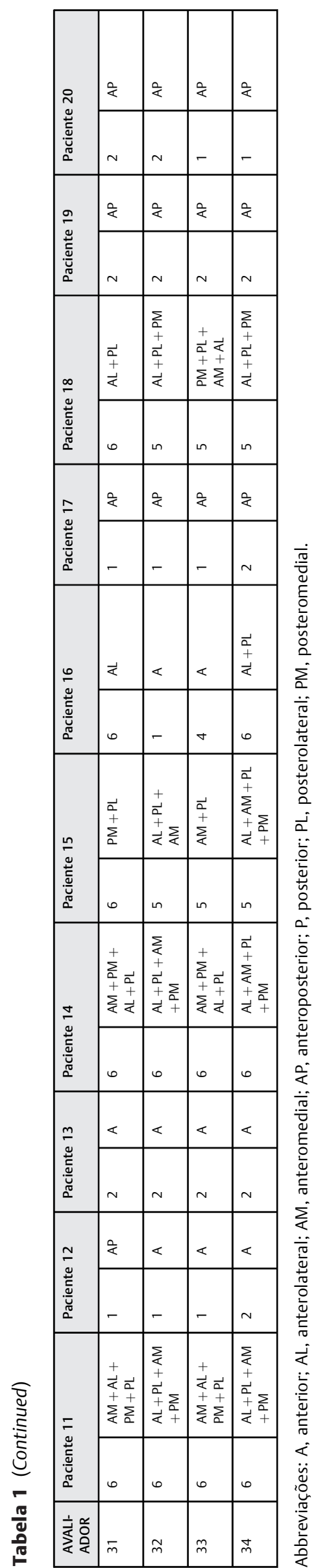

Tabela 2 Concordância interobservador $(n=34)$ dos sistemas de classificação de Schatzker e da modificação de Kfuri para 20 fraturas do planalto tibial

\begin{tabular}{|l|l|l|l|}
\hline \multirow{2}{*}{ Classificação } & \multicolumn{3}{|l|}{ Concordância inter-observador (к) } \\
\cline { 2 - 4 } & Geral & \multicolumn{3}{|l|}{ Nível de experiência } \\
\cline { 2 - 4 } & & Especialistas & Residentes \\
\hline Schatzker & 0.46 & 0.52 & 0.45 \\
\hline $\begin{array}{l}\text { Modificação } \\
\text { de Kfuri }\end{array}$ & 0.30 & 0.39 & 0.28 \\
\hline
\end{tabular}

К - Kappa de Fleiss.

tomográficas e radiográficas para classificar pelo sistema de Schatzker. A importância do uso da TC para aumentar a confiabilidade da classificação de Schatzker ainda não está clara. ${ }^{25}$ Alguns trabalhos investigaram a concordância somente incluindo imagens de RX, com resultados semelhantes. ${ }^{8,9,13,22}$ Um estudo avaliando 50 fraturas do planalto tibial somente com RX e, posteriormente, com RX e TC, mostrou que não houve diferenças em relação à reprodutibilidade. ${ }^{23}$ Entretanto, outros trabalhos mostraram aumento significativo dos valores de Kappa para o diagnóstico das fraturas após a inclusão da TC, principalmente imagens em 3D. ${ }^{15,21,25,26}$ Um estudo recente investigou o efeito da adição das imagens tomográficas em 3D na classificação de fraturas do planalto tibial, concluindo que o acréscimo da terceira dimensão não melhorou a reprodutibilidade do sistema; na verdade, o uso das imagens em 2D isoladamente tiveram concordância mais elevada. ${ }^{14}$ Apesar do papel da TC na concordância inter- e intraobservador das fraturas do planalto tibial ainda necessitar ser determinado, ${ }^{24}$ essa ferramenta mostrouse fundamental na escolha da via de acesso cirúrgico, principalmente nas lesões com orientação no plano coronal. ${ }^{15,27}$

Estudos prévios avaliando as fraturas do planalto tibial relataram que a experiência dos observadores não influenciou o grau de confiabilidade das classificações. . $^{93,14,21}$ Interessantemente, no presente estudo com 34 observadores, sendo 24 "especialistas" e 10 residentes, observamos que a concordância interobservador foi superior entre os menos experientes $(\mathrm{K}=$ 0.52 e 0.39 ) em relação aos especialistas $(K=0.45$ e 0.28$)$, para a classificação de Schatzker e a modificação de Kfuri, respectivamente. A modificação de Kfuri foi recentemente publicada, ainda não sendo amplamente difundida nem mesmo entre os especialistas. Além disso, a explanação feita pelos autores previamente à avaliação pelos participantes pode ter deixado dúvidas sobre a correta aplicação do sistema, fato que pode justificar a maior concordância entre os residentes que recorreram mais vezes às figuras ilustrativas. Corroborando nossa hipótese, Sacramento et al. ${ }^{20}$ estudaram pacientes com fratura do tornozelo, demonstrando que residentes tiveram maior concordância inter- e intraobservador para as classificações de Danis-Weber, de Lauge-Hansen e do grupo A.O. (Arbeitsgemeinschaft für Osteosynthesefragen), sugerindo que os observadores menos experientes provavelmente utilizaram mais as imagens das classificações disponibilizadas como gabarito.

Estamos cientes das limitações que o presente estudo apresenta. Dentre elas, podemos citar a falta de avaliação da concordância intraobservadores e da avaliação da concordância dos 
diferentes exames de imagem, RX e TC, separadamente. Além disso, o número de observadores e de casos/fraturas estudadas diferiu da maioria dos trabalhos previamente publicados, em especial do único estudo que avaliou o sistema proposto por Kfuri, ${ }^{15}$ que incluiu 70 casos avaliados por 10 observadores. 0 tamanho da amostra pode ter sido um fator importante para a baixa concordância observada, uma vez que esta pode ter subestimado a heterogeneidade dos padrões de fratura possíveis para o planalto tibial. Por último, disponibilizamos somente parte das imagens da TC; assim, como o observador não pôde avaliar o conjunto inteiro de imagens, o corte apresentado poderia não ser totalmente representativo da fratura. Entretanto, em um estudo recente, os avaliadores tiveram acesso a vídeos das TCs em 2D e 3D, apresentando valores de concordância inferiores aos nossos para a classificação de Schatzker. ${ }^{14}$

\section{Conclusão}

A classificação de Schatzker e a classificação modificada por Kfuri apresentaram concordância interobservadores moderada e leve, respectivamente. Além disso, os residentes apresentaram concordâncias superiores aos especialistas para os dois sistemas estudados.

\section{Declaração de Contribuição dos Autores}

Cada autor contribuiu individual e significantemente para o desenvolvimento do presente artigo. Mansur H. (00000001-7527-969X): coleta de dados, análise dos dados, redação e revisão do artigo, todo conceito intelectual do artigo, confecção de todo o projeto de pesquisa; VLBC (0000-0003-3236-4813): coleta de dados, redação do artigo e revisão do artigo; Abdo B.(0000-0001-5269-9106): coleta de dados, análise dos dados; Ramos L. S. (00000002-7233-796X): redação do artigo e revisão do artigo; Castiglia M. T. (0000-0001-7543-2199): revisão do artigo.

\section{Suporte Financeiro}

Não houve suporte financeiro de fontes públicas, comerciais, ou sem fins lucrativos.

\section{Conflito de Interesses}

Os autores declaram não haver conflito de interesses.

\section{Referências}

1 Jacofsky DJ, Haidukerwych GJ. Tibia plateau fractures. In: Scott WN. Insall \& Scott Surgery of the knee. $4^{\text {th }}$ ed. Philadelphia: Churchill Livingstone; 2006:1133-1146

2 Watson JT, Schatzker J. Tibial plateau fractures. In: Browner BD, Jupiter JB, Levine AM, Trafton PG, editors. Skeletal trauma. Basic science, management, and reconstruction Philadelphia: Saunders; 2003:2074-2130

3 Thomas Ch, Athanasiov A, Wullschleger M, Schuetz M. Current concepts in tibial plateau fractures. Acta Chir Orthop Traumatol Cech 2009;76(05):363-373

4 Tscherne H, Lobenhoffer P. Tibial plateau fractures. Management and expected results. Clin Orthop Relat Res 1993;(292):87-100

5 Junior MK, Fogagnolo F, Bitar RC, Freitas RL, Salim R, Paccola CJA. Fraturas do planalto tibial. Rev Bras Ortop 2009;44(06):468-474

6 Millar SC, Arnold JB, Thewlis D, Fraysse F, Solomon LB. A systematic literature review of tibial plateau fractures: What classifi- cations are used and how reliable and useful are they? Injury 2018;49(03):473-490

7 Pires e Albuquerque R, Giordano V, Pallottino A, et al. Análise da reprodutibilidade das classificações das fraturas do platô tibial. Rev Bras Ortop 2009;44(03):225-229

8 Maripuri SN, Rao P, Manoj-Thomas A, Mohanty K. The classification systems for tibial plateau fractures: how reliable are they? Injury 2008;39(10):1216-1221

9 Charalambous CP, Tryfonidis M, Alvi F, et al. Inter- and intraobserver variation of the Schatzker and AO/OTA classifications of tibial plateau fractures and a proposal of a new classification system. Ann R Coll Surg Engl 2007;89(04):400-404

10 Mandarino M, Pessoa A, Guimarães JAM. Avaliação da reprodutibilidade da classificação de Schatzker para as fraturas do planalto tibial. Rev Into 2004;2(02):11-18

11 Schatzker J. Compression in the surgical treatment of fractures of the tibia. Clin Orthop Relat Res 1974;(105):220-239

12 Kfuri M, Schatzker J. Revisiting the Schatzker classification of tibial plateau fractures. Injury 2018;49(12):2252-2263

13 Walton NP, Harish S, Roberts C, Blundell C. AO or Schatzker? How reliable is classification of tibial plateau fractures?. Arch Orthop Trauma Surg 2003;123(08):396-398

14 Mellema JJ, Doornberg JN, Molenaars RJ, Ring D, Kloen PTraumaplatform Study Collaborative \& Science of Variation Group. Interobserver reliability of the Schatzker and Luo classification systems for tibial plateau fractures. Injury 2016;47(04):944-949

15 Castiglia MT, Nogueira-Barbosa MH, Messias AMV, et al. The Impact of Computed Tomography on Decision Making in Tibial Plateau Fractures. J Knee Surg 2018;31(10):1007-1014

16 Fleiss JL. Measuring nominal scale agreement among many raters. Psychol Bull 1971;76(05):378-382

17 Viera AJ, Garrett JM. Understanding interobserver agreement: the kappa statistic. Fam Med 2005;37(05):360-363

18 Utino AY, de Alencar DR, Fernadez Maringolo L, Negrão JM, Blumetti FC, Dobashi ET. Intra and interobserver concordance of the AO classification system for fractures of the long bones in the pediatric population. Rev Bras Ortop 2015;50(05):501-508

19 Landis JR, Koch GG. The measurement of observer agreement for categorical data. Biometrics 1977;33(01):159-174

20 Ramos LS, Gonçalves HM, Freitas A, Oliveira MP, Lima DMS, Carmargo WS. Avaliação da reprodutibilidade das classificações Lauge-Hansen, Danis-Weber e AO para fraturas do tornozelo. . [Publicado on-line: 2020-12-18] Rev Bras Ortop. Disponível emhttps://www.thieme-connect.com/products/ejournals/abstract/10.1055/s-0040-1718508

21 Taşkesen A, Demirkale İ, Okkaoğlu MC, Özdemir M, Bilgili MG Altay M. Intraobserver and interobserver reliability assessment of tibial plateau fracture classification systems. Eklem Hastalik Cerrahisi 2017;28(03):177-181

22 Zhu Y, Hu CF, Yang G, Cheng D, Luo CF. Inter-observer reliability assessment of the Schatzker, AO/OTA and three-column classification of tibial plateau fractures. J Trauma Manag Outcomes 2013;7(01):7

23 Gicquel T, Najihi N, Vendeuvre T, Teyssedou S, Gayet LE, Huten D. Tibial plateau fractures: reproducibility of three classifications (Schatzker, AO, Duparc) and a revised Duparc classification. Orthop Traumatol Surg Res 2013;99(07):805-816

24 de Lima Lopes C, da Rocha Cândido Filho CA, de Lima E Silva TA, Gonçalves MC, de Oliveira RL, de Lima PR. Importance of radiological studies by means of computed tomography for managing fractures of the tibial plateau. Rev Bras Ortop 2014;49(06):593-601

25 Brunner A, Horisberger M, Ulmar B, Hoffmann A, Babst R. Classification systems for tibial plateau fractures; does computed tomography scanning improve their reliability? Injury 2010;41(02):173-178

26 Rafii M, Lamont JG, Firooznia H. Tibial plateau fractures: CT evaluation and classification. Crit Rev Diagn Imaging 1987;27(02):91-112

27 Kfuri M, Schatzker J, Castiglia MT, Giordano V, Fogagnolo F, Stannard JP. Extended Anterolateral Approach for Complex Lateral Tibial Plateau Fractures. J Knee Surg 2017;30(03):204-211 